\title{
Ultrafast intersystem crossing in xanthone from wavepacket dynamics simulations
}

\author{
Marc Alías-Rodríguez, ${ }^{1}$ Coen de Graaf, ${ }^{1,2}$ and Miquel Huix-Rotllant ${ }^{3, \text { a) }}$ \\ 1) Departament de Química Física i Inorgànica, Universitat Rovira i Virgili, Marcel.lí Domingo 143007 Tarragona, \\ Catalunya, Spain. \\ 2) ICREA, Passeig Lluís Companys 23, Barcelona, Spain \\ ${ }^{3)}$ Aix-Marseille Univ, CNRS, ICR, Marseille, France.
}

\begin{abstract}
Most aromatic ketones containing first-row elements undergo unexpectedly fast intersystem crossing in few tens of picoseconds and a quantum yield close to unity. Among them, xanthone (9H-xanthen-9-one) possesses one of the fastest intersystem crossing rates of $\sim 1.5 \mathrm{ps}$, despite containing only first-row elements. The exact mechanism of this unusually fast singlet-triplet transition is still under debate. Here, we perform a complete wavepacket dynamics simulation of the internal conversion and intersystem crossing reactions of xanthone in the gas phase. We show that xanthone follows El-Sayed's rule for intersystem crossing. From the second singlet excited state, the mechanism is sequential: (i) an internal conversion between $\operatorname{singlets}{ }^{1} \pi \pi^{*} \rightarrow{ }^{1} n \pi^{*}$ (85 fs), (ii) an intersystem crossing ${ }^{1} n \pi^{*} \rightarrow{ }^{3} \pi \pi^{*}$ (2.0 ps), and (iii) an internal conversion between triplets ${ }^{3} \pi \pi^{*} \rightarrow{ }^{3} n \pi^{*}$ (602 fs). Each transfer finds its origin in a barrierless access to electronic state intersections. These intersections are close to minimum energy structures, allowing for efficient radiationless transitions from the initial singlet state to the triplets.
\end{abstract}

Aromatic ketones are well known for their phosphorescent properties due to an efficient intersystem crossing (ISC) $)^{1-4}$ non-radiative transfer between singlet and triplet excited states of the type $n \pi^{*}$ and $\pi \pi^{*}$. The $n$ orbital corresponds to the carbonyl lone pair orbital mixed with $\sigma$ orbitals on the carbon bonds, while the $\pi$ and $\pi^{*}$ are a mixture of bonding and anti-bonding combinations of the atomic $\mathrm{p}_{z}$ orbitals centred on the carbonyl and the aromatic rings. According to classical photochemistry rules for ISC (known as El-Sayed's rules), ${ }^{5}$ an efficient transition is characterized by simultaneous strong spin-orbit coupling and a small energetic gap between singlet and triplets states of dissimilar character, that is, ${ }^{1} n \pi^{*} \rightarrow{ }^{3} \pi \pi^{*}$ or ${ }^{1} \pi \pi^{*} \rightarrow{ }^{3} n \pi^{*}$. The lifetime of triplet states in aromatic ketones are reported to last from few to several tens of picoseconds, depending mainly on the energetic gap between $n \pi^{*}$ and $\pi \pi^{*}$ states. For example, acetophenone ISC rate is reported to be $42 \mathrm{ps},{ }^{6}$ benzophenone $5-10 \mathrm{ps},{ }^{7}$, anthrone $70 \mathrm{ps},{ }^{8}$ etc. This gap can be modulated or even interchanged singlet and triplet depending on the type of ketone, ${ }^{8-11}$ solvent polarity, ${ }^{12-16}$ temperature, ${ }^{2}$ pressure, ${ }^{17}$ substituents, ${ }^{16,18}$ concentration, ${ }^{19}$ etc.

It is currently widely accepted that vibrations play a central role in modulating the efficiency of non-radiative decays, ${ }^{20}$ and intersystem crossings of aromatic ketones are no exception. ${ }^{21-24}$ One of us performed one of the first wavepacket dynamics simulations in acetophenone, showing a rapid ${ }^{3} n \pi^{*} /{ }^{3} \pi \pi^{*}$ internal conversion after the ${ }^{1} n \pi^{*} \rightarrow{ }^{3} \pi \pi^{*}$ intersystem crossing. ${ }^{23}$ Almost simultaneously, Granucci and coworkers performed a study of benzophenone using fewest-switch surface hopping, obtaining a $16 \mathrm{ps}$ direct transfer from the lowest singlet to the lowest triplet, due to the mixed $n \pi^{*}$ and $\pi \pi^{*}$ char-

a) Electronic mail: miquel.huixrotllant@univ-amu.fr acter of the states. ${ }^{24}$ Marian and coworkers have studied the ISC of thioxanthone ${ }^{25-27}$ and xanthone ${ }^{22}$ using Fermi Golden's rule to determine vibronic intersystem crossing rates.

The triplet quantum yield of xanthone in solution is close to unity. ${ }^{12}$ Among the aromatic ketones, xanthone has one of the fastest intersystem crossings, estimated to be around 1-1.5 picoseconds in ethanol by femtosecond fluorescent experiments. ${ }^{28-30}$ In these experiments, two kinetic steps of $1.5 \mathrm{ps}$ and $12 \mathrm{ps}$ were obtained. Two mechanisms were proposed to explain these rates: (i) sequential mechanism, with a fast ${ }^{1} \pi \pi^{*} \rightarrow{ }^{3} n \pi^{*}$ transfer in $1.5 \mathrm{ps}$ and a slow component of $12 \mathrm{ps}$ belonging to the internal conversion between triplets, and (ii) a branching mechanism, in which the 1.5 ps component corresponds to both the ISC ${ }^{1} \pi \pi^{*} \rightarrow{ }^{3} n \pi^{*}$ and the internal conversion ${ }^{1} \pi \pi^{*} \rightarrow{ }^{1} n \pi^{*}$, and the slow 12 ps component to the ${ }^{1} n \pi^{*} \rightarrow{ }^{3} \pi \pi^{*}$ transfer from the lowest singlet. Rai-Constapel and coworkers simulated the intersystem crossing kinetic rate in gas phase and solution of xanthone using Fermi Golden's rule. ${ }^{22}$ In the gas phase, they obtained a fast ${ }^{1} \pi \pi^{*} \rightarrow{ }^{3} \pi \pi^{*}$ ISC of about $5 \mathrm{ps}$, and a ${ }^{1} \pi \pi^{*} \rightarrow{ }^{3} n \pi^{*}$ ISC of $200 \mathrm{ps}$, in apparent contradiction with El-Sayed's rule. As for the ${ }^{1} n \pi^{*} \rightarrow{ }^{3} \pi \pi^{*}$ ISC rate of $0.13 \mathrm{ps}$, which is $10-100$ times faster than the experimental reported values. The ${ }^{1} n \pi^{*} \rightarrow{ }^{3} n \pi^{*}$ transition was estimated at ca. 330 ps. Recently, Bracker et al. extended the use of Fermi's golden rule for both the internal coversion and the intersystem crossing. ${ }^{31}$ They results suggested that ${ }^{1} \pi \pi^{*}{ }_{-}^{3} \pi \pi^{*}$ ISC and ${ }^{1} \pi \pi^{*}{ }_{-}{ }^{1} \mathrm{n} \pi^{*}$ IC to be competitive transitions and proposed a unified branched mechanism.

Here, we report a quantum wavepacket dynamics of xanthone including the lowest two singlet and the four triplet excited states. The model contains all nonadiabatic and spin-orbit couplings to represent the internal conversion and intersystem crossing on the same 


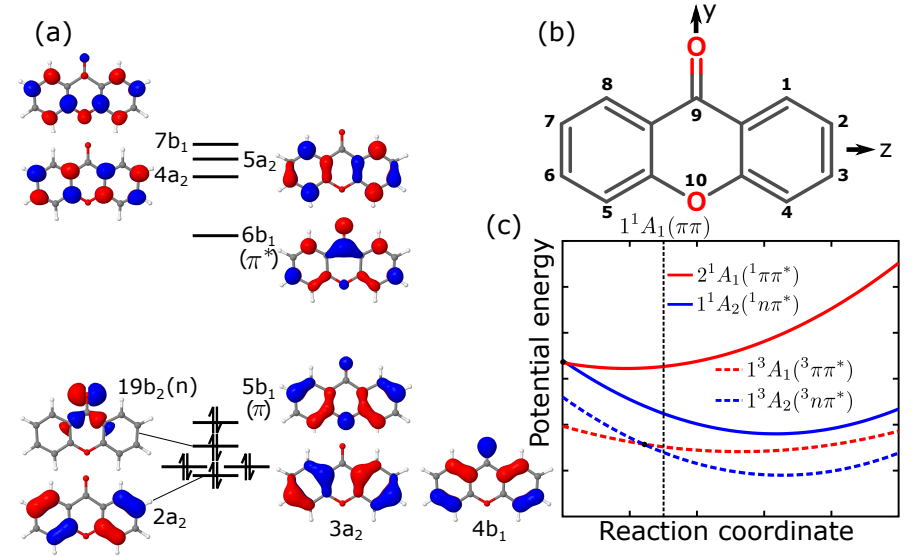

FIG. 1. Schematic structure of 9H-xanthen-9-one (xanthone) with the Cartesian axis orientation and the ring atom's numbering. On the left, the 5 highest occupied and 4 lowest unoccupied orbitals are shown tagged by the symmetry label of $\mathrm{C}_{2 v}$, and the short-hand notation in parenthesis. On the right, schematic diabatic potential energy surface of the two lowest singlet (solid line) and triplet (dashed line) excited states for the $n \pi^{*}$ (red) and $\pi \pi^{*}$ states (blue), where the vertical dashed line represents the Franck-Condon geometry.

footing, including the effect of Duschinsky rotations. ${ }^{32}$ The total vibronic Hamiltonian can be written as

$$
\mathbf{H}(\mathbf{Q})=[\mathbf{T}(\mathbf{Q})+\mathbf{V}(\mathbf{Q})] \mathbf{1}+\mathbf{H}^{\mathrm{NA}}(\mathbf{Q})+\mathbf{H}^{\mathrm{SO}}(\mathbf{Q}) \text {. }
$$

Here, $\mathbf{T}(\mathbf{Q})$ and $\mathbf{V}(\mathbf{Q})$ are the kinetic and diabatic potential energies accounting for the reorganization energy in each electronic state, and $\mathbf{H}^{\mathrm{NA}}(\mathbf{Q})$ and $\mathbf{H}^{\mathrm{SO}}(\mathbf{Q})$ are the vibronic non-adiabatic and spin-orbit couplings respectively representing the non-radiative internal conversion and intersystem crossings respectively. The explicit expressions for the Hamiltonian blocks and the parametrization are described in Sec. S1 of the supporting information.

Xanthone lowest energy state is a singlet $1^{1} \mathrm{~A}_{1}$ state (using $\mathrm{C}_{2 v}$ point group symmetry labeling, with the orientation as described in Fig. 1). At this geometry, the $1^{1} \mathrm{~A}_{2}$ state is the first excited singlet, corresponding to a $19 \mathrm{~b}_{2} \rightarrow 6 \mathrm{~b}_{1}$ transition (also called ${ }^{1} n \pi^{*}$ state), which is dipole-forbidden. The second singlet excited state is the $2^{1} \mathrm{~A}_{1}$ state, which is represented by a $5 \mathrm{~b}_{1} \rightarrow 6 \mathrm{~b}_{1}$ transition (more commonly referred to ${ }^{1} \pi \pi^{*}$ state), and is dipoleallowed. The vibrationally resolved absorption spectrum in gas phase for ${ }^{1} \pi \pi^{*}$ is shown in Fig. 2 and compared to the experimental spectrum in gas phase taken from Ref. 33. The theoretical spectrum has been obtained by the Fourier transform of the autocorrelation function extracted from a wavepacket propagation on the ${ }^{1} \pi \pi^{*}$ state with the Hamiltonian defined in Eq. 1, that is, considering the spin-orbit and vibronic couplings of ${ }^{1} \pi \pi^{*}$ state. ${ }^{34,35}$ For analysis purposes, we have performed a vibrational integral overlap computation using the FranckCondon and Herzberg-Teller approximations for the diabatic $\mathrm{S}_{0} \rightarrow{ }^{1} \pi \pi^{*}$ transition. ${ }^{36,37}$ The most intense peak is the fundamental zero-zero transition $\left|0^{0}\right\rangle \rightarrow\left|0^{0}\right\rangle$, fol-

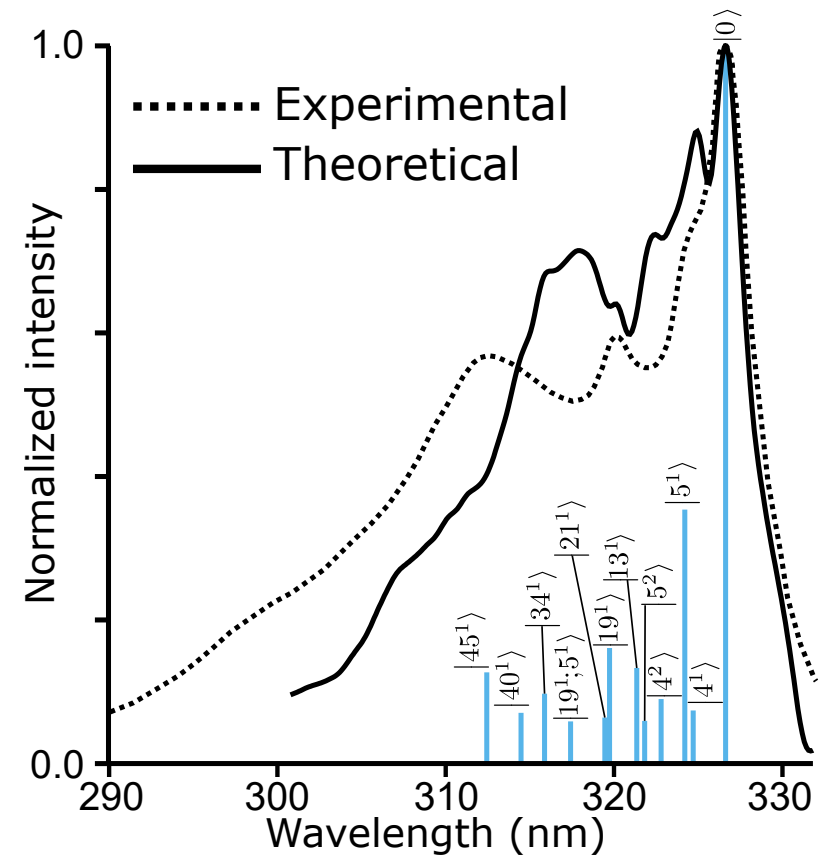

FIG. 2. Comparison of the experimental and theoretical gas phase singlet-singlet absorption spectra of xanthone for the $2^{1} \mathrm{~A}_{1}$ state $\left({ }^{1} \pi \pi^{*}\right)$ transition. Experimental data has been extracted from Ref. 33, and corresponds to the gas phase UV spectrum of xanthone recorded at $150{ }^{\circ} \mathrm{C}$. The solid line corresponds to the Fourier transform of the numerical autocorrelation function obtained during wavepacket propagation. For analysis purposes, the stick spectrum is shown as blue vertical lines with their corresponding analys, corresponding to the Franck-Condon Herzberg-Teller vibrational expansion. All theoretical spectra have been shifted and normalized to the most intense peak of the experimental spectrum.

lowed by two fundamental and two overtone transitions to $|4\rangle$ (mode frequency of $231.8 \mathrm{~cm}^{-1}, \mathrm{a}_{1}$, in-plane) and |5) $\left(247.3 \mathrm{~cm}^{-1}, \mathrm{a}_{2}\right.$, out-of-plane $)$ that show up as a shoulder next to the main transition. The next vibrational transitions is represented by fundamental transitions to $|13\rangle\left(530.0 \mathrm{~cm}^{-1}, \mathrm{a}_{2}\right.$, coupling mode $),|19\rangle(713.6$ $\mathrm{cm}^{-1}, \mathrm{a}_{1}$, tuning mode) and $|21\rangle\left(773.3 \mathrm{~cm}^{-1}, \mathrm{~b}_{1}\right.$, tuning mode), while the last vibrational peak is essentially represented by fundamental transitions to $|34\rangle$ (1060.0 $\mathrm{cm}^{-1}, \mathrm{~b}_{2}$, tuning mode), $|40\rangle\left(1248.8 \mathrm{~cm}^{-1}\right.$, $\mathrm{a}_{1}$, tuning mode) and $|45\rangle\left(1379.0 \mathrm{~cm}^{-1}, \mathrm{~b}_{2}\right.$, tuning mode $)$.

In the minimum energy structure of $1^{1} \mathrm{~A}_{1}$ state (Fig. 1 ), the triplets $1^{3} \mathrm{~A}_{2}$ and $1^{3} \mathrm{~A}_{1}$ are quasi-degenerate (0.03 $\mathrm{eV}$ gap), $0.166 \mathrm{eV}$ below the singlet $1^{1} \mathrm{~A}_{2}$ state, in good agreement with the experimental value of $0.14 \mathrm{eV} .^{38}$ The $1^{1} \mathrm{~A}_{2}$ state is only $0.06 \mathrm{eV}$ below the $2^{3} \mathrm{~A}_{1}$ described mainly by a $4 \mathrm{~b}_{1} \rightarrow 6 \mathrm{~b}_{1}$ transition, and the triplet $1^{3} \mathrm{~B}_{2}$ described mainly by a $3 \mathrm{a}_{2} \rightarrow 6 \mathrm{~b}_{1}$ is found $\sim 0.11 \mathrm{eV}$ above $1^{1} \mathrm{~A}_{2}$. The $2^{1} \mathrm{~A}_{1}$ state $\left(5 \mathrm{~b}_{1} \rightarrow 6 \mathrm{~b}_{1}\right)$ is the highest energy state, $0.4 \mathrm{eV}$ above the $1^{1} \mathrm{~A}_{2}$ state. Minimal energy structures of the lowest singlet and triplet states are planar of quasi- $\mathrm{C}_{2 v}$ symmetry. As for the state crossings, on the one hand the $2^{1} \mathrm{~A}_{1} / 1^{1} \mathrm{~A}_{2}\left({ }^{1} \pi \pi^{*} /{ }^{1} n \pi^{*}\right)$ cross in 
an accidental different symmetry structure of $\mathrm{C}_{s}$ resulting from the linear combination of the carbonyl symmetric stretching (essentially of $\mathrm{a}_{1}$ symmetry) and an asymmetric stretching vibration of mostly $b_{1}$ symmetry involving the carbon atoms on the aromatic ring. On the other hand, $1^{1} \mathrm{~A}_{2}$ crosses with $1^{3} \mathrm{~A}_{1}$ state in a quasi- $\mathrm{C}_{2 v}$ structure expanded through totally symmetric vibrations involving the carbonyl symmetric stretching of the aromatic rings.

Non-zero non-adiabatic coupling of $A_{1}$ and $A_{2}$ states is only occurring in distorted geometries whose point group symmetry is lowered to $\mathrm{C}_{2}$ sub-group by means of asymmetric out-of-plane $a_{2}$ vibrations (hereafter referred as "coupling modes"). This indeed mixes $n \pi^{*}$ and $\pi \pi^{*}$ states as was shown previously for benzophenone. ${ }^{24}$ None of the minimum energy structures or minimum energy crossing points belongs to the $\mathrm{C}_{2}$ subgroup, and thus, only accidental different symmetry intersections are happening in xanthone. The $b_{1}$ modes are out-of-plane symmetric distortions, breaking the symmetry to the $\mathrm{C}_{s}$ sub-group in which $\sigma_{x y}$ is the plane of symmetry. The lone pair orbital $\left(19 b_{2}\right)$ becomes a", while the $\pi$ orbitals of $b_{1}$ symmetry become a', and thus $n \pi^{*}$ and $\pi \pi^{*}$ are uncoupled. These modes are not introducing couplings among the $\mathrm{A}_{2}$ and $A_{1}$ states. The "tuning modes", that is, the modes that modulate the gap and describe the reorganization energy of the excited states, are of $b_{2}$ symmetry (asymmetric inplane) and $a_{1}$ symmetry modes (symmetric in-plane). In $\mathrm{a}_{1}$, the $\mathrm{C}_{2 v}$ point group symmetry is maintained, whereas a symmetry breaking $\mathrm{C}_{s}$ sub-group in which $\sigma_{y z}$ (that is, the molecular plane) is the plane of symmetry is observed along $\mathrm{b}_{2}$ symmetry modes. Similar to $\mathrm{b}_{1}, \mathrm{n}$ - and $\pi$-type orbitals fall in different symmetries and thus are uncoupled.

The spin-orbit coupling follows different symmetry rules than the non-adiabatic couplings. The singlet $2^{1} \mathrm{~A}_{1}$ state mixes with triplets $1^{3} \mathrm{~A}_{2}$ and $1^{3} \mathrm{~B}_{2}$, but not to other $1^{3} \mathrm{~A}_{1}$ or $2^{3} \mathrm{~A}_{1}$ states. However, spin-vibronic coupling can activate the mixing with $A_{1}$ triplets through vibrations of $a_{2}, b_{1}$ and $b_{2}$ type. The $1^{1} A_{2}$ on the other hand couples to triplets $1^{3} \mathrm{~A}_{1}, 2^{3} \mathrm{~A}_{1}$ and $1^{3} \mathrm{~B}_{2}$, and can couple to the $1^{3} \mathrm{~A}_{2}$ through vibronic spin-orbit with vibrations of the type $a_{2}, b_{1}$ and $b_{2}$ type. Still, the vibronic spinorbit couplings are small and thus intersystem crossing can happen only between states that are energetically close in energy. The $2^{1} \mathrm{~A}_{1}$ is energetically far from the rest of the triplet manifold, while the $1^{1} \mathrm{~A}_{2}$ state is energetically close to triplets of different symmetry. Thus, the latter singlet is expected to be the main state from which intersystem crossing happens for xanthone in the gas phase.

The dynamic evolution of diabatic populations is shown in Fig. 3. The simulations have been started at the $2^{1} \mathrm{~A}_{1}$ state. The population of this state rapidly decays in the first $200 \mathrm{fs}$, concomitant to the $1^{1} \mathrm{~A}_{2}$ population rise, indication of the fast internal conversion between the two states. This is due to the barrierless access to two $1^{1} \mathrm{~A}_{2} / 2^{1} \mathrm{~A}_{1}$ accidental different symmetry intersec-

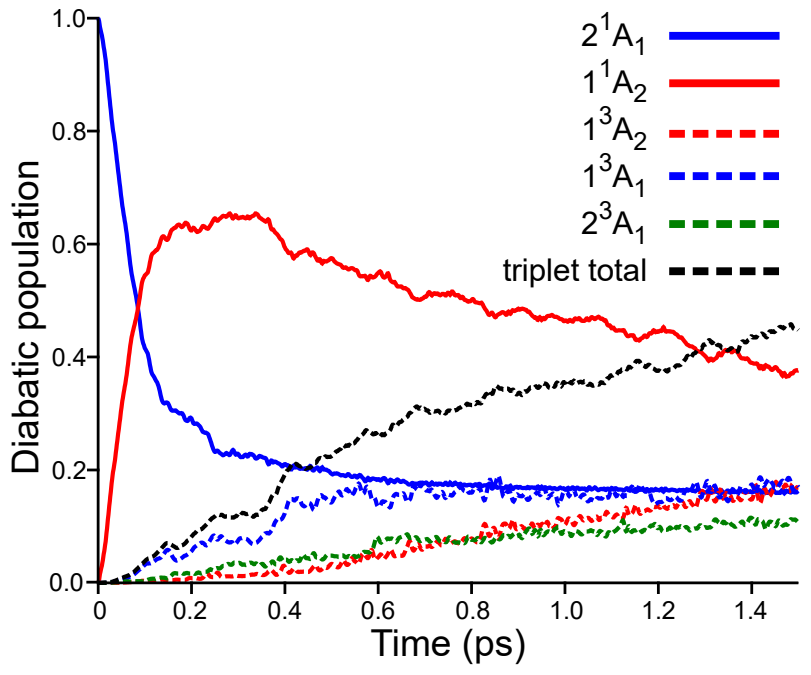

FIG. 3. Evolution of diabatic populations for the singlets $2^{1} \mathrm{~A}_{1}\left({ }^{1} \pi \pi^{*}\right.$, solid blue) and $1^{1} \mathrm{~A}_{2}\left({ }^{1} n \pi^{*}\right.$, solid red $)$, and triplets $1^{3} \mathrm{~A}_{2}\left({ }^{3} n \pi^{*}\right.$, dashed red $), 1^{3} \mathrm{~A}_{1}\left({ }^{3} \pi \pi^{*}\right.$, dashed blue) and $2^{3} \mathrm{~A}_{1}$ (dashed green). The black dashed line corresponds to the sum of all triplet population. The dynamics have been started from the $2^{1} \mathrm{~A}_{1}$ state.

tions. In this process, $80 \%$ of the $2^{1} \mathrm{~A}_{1}$ is transferred to $1^{1} A_{2}$. Still, $20 \%$ of the population remains trapped in $2^{1} \mathrm{~A}_{1}$, and decays at a slower rate. The $1^{1} \mathrm{~A}_{2}$ being the most populated state after 200 fs rapidly decays to the triplets. Initially, the intersystem crossing is mainly due to the $1^{1} \mathrm{~A}_{2} \rightarrow 1^{3} \mathrm{~A}_{1}$ transition and to a lesser extent $1^{1} \mathrm{~A}_{2} \rightarrow 2^{3} \mathrm{~A}_{1}$. These are the only contributions to the build up of triplet populations, consistent thus with El-Sayed's rule for intersystem crossing. The transfer between triplets slowly builds up the population on the lowest triplet $1^{3} \mathrm{~A}_{2}$.

For interpretation purposes, a kinetic mechanism can be inferred from the 1.5 ps population dynamics of Fig. 3 , by fitting it to a first-order kinetic model (see Fig. S7 in the Supporting Information), taking into account that kinetic rates beyond the propagated time are extrapolated and thus less reliable. From the model, we can infer that the mechanism follows mainly a sequential transfer: $2^{1} \mathrm{~A}_{1} \stackrel{\mathrm{IC}}{\longrightarrow} 1^{1} \mathrm{~A}_{2} \stackrel{\text { ISC }}{\longrightarrow} 1^{3} \mathrm{~A}_{1} \stackrel{\text { IC }}{\longrightarrow} 1^{3} \mathrm{~A}_{2}$. First, the kinetics of $2^{1} \mathrm{~A}_{1}$ state decays biexponentially to the $1^{1} \mathrm{~A}_{2}$ state via internal conversion. Indeed, $80 \%$ of the population of the second singlet excited state decays in a fast rate of $85 \mathrm{fs}$. This fast rate is interpreted as the first time the wavepacket reaches the $2^{1} \mathrm{~A}_{1} / 1^{1} \mathrm{~A}_{2}$ crossing with a large momentum, since the path to it is barrierless. As explained, this crossing is an accidental different symmetry crossing, and thus, only activation of coupling modes of $a_{2}$ symmetry can make this transition happen, which could explain the incomplete transfer. After that, $15 \%$ of the wavepacket remains trapped in the $2^{1} \mathrm{~A}_{1}$ minimum, and slowly decays to $1^{1} \mathrm{~A}_{2}$ with a rate of 5 ps via the activation of coupling modes. In the $1^{1} \mathrm{~A}_{2}$ state, there are two possible ISC transfers. The $1^{1} \mathrm{~A}_{2} \rightarrow 2^{3} \mathrm{~A}_{1}$ and 
$1^{1} \mathrm{~A}_{2} \rightarrow 1^{3} \mathrm{~B}_{2}$ triplet rates are obtained at 6.73 and 44.64 respectively ps. In this time, we do not observe a fast internal conversion to the $1^{3} \mathrm{~A}_{1}$ and $1^{3} \mathrm{~A}_{2}$, but probably this occurs at longer times. The fastest important ISC is the $1^{1} \mathrm{~A}_{2} \rightarrow 1^{3} \mathrm{~A}_{1}$, which is the first populated triplet in time. The rate for this transfer is given by $2.0 \mathrm{ps}$, which is probably explained by a strong spin-orbit coupling and the fact that the ISC minimum energy crossing geometry is close to the minimum of $1^{1} \mathrm{~A}_{2}$ state. The internal conversion between $1^{3} \mathrm{~A}_{1} \leftrightarrow 1^{3} \mathrm{~A}_{2}$ is the only pathway we obtained of population of the lowest triplet state, and is estimated at $602 \mathrm{fs}$ for the transfer to $1^{3} \mathrm{~A}_{2}$ and $803 \mathrm{fs}$ for the back transfer to $1^{3} \mathrm{~A}_{1}$.

In the literature, the possibility of a major channel corresponding to a "non-Kasha" transfer directly from $2^{1} \mathrm{~A}_{1}$ to the $1^{3} \mathrm{~A}_{2}$ triplet was evoked, first by Baba and coworkers for xanthone in the gas phase,${ }^{38,39}$ and later confirmed by Gilch and coworkers for xanthone in ethanol ${ }^{28,29}$. Baba et al. estimated the rate of transfer from $2^{1} \mathrm{~A}_{1}$ in the gas phase at ca. $100 \mathrm{fs},{ }^{39}$ while the experiments of Gilch et al. in ethanol estimated it at $1.5 \mathrm{ps}^{28}$ Recently, theoretical studies using rate theory in the gas phase by Rai-Constapel and coworkers predicted a 5 ps for the $2^{1} \mathrm{~A}_{1} \rightarrow 1^{3} \mathrm{~A}_{1}$ (El-Sayed's forbidden transition), while the $2^{1} \mathrm{~A}_{1} \rightarrow 1^{3} \mathrm{~A}_{2}$ (El-Sayed's allowed transition) was predicted to be at a rate of $200 \mathrm{ps}^{22}$ In the gas phase, the main decay channel observed from $2^{1} \mathrm{~A}_{1}$ is to the $1^{1} \mathrm{~A}_{2}$. This is estimated at a rate of $135 \mathrm{fs}$, close to the experimental decay of $100 \mathrm{fs}$ observed for $2^{1} \mathrm{~A}_{1}$ in the gas phase. ${ }^{39}$ This is due to a barrierless access to the conical intersection region between the two states. The $2^{1} \mathrm{~A}_{1}$ state has an almost negligible vibronic spin-orbit coupling with energetically close $2^{3} \mathrm{~A}_{1}$ and $1^{3} \mathrm{~B}_{2}$ states (see Table $\mathrm{S} 7$ in the supporting information). The $2^{3} \mathrm{~A}_{1}$ triplet receives $5-10 \%$ of the population, while the population to the $1^{3} \mathrm{~B}_{2}$ states is negligible. This could explain the triplet coupling evoked for the $2^{3} \mathrm{~A}_{1}$ state. ${ }^{38}$ No direct transfer from $2^{1} \mathrm{~A}_{1}$ is observed to $1^{3} \mathrm{~A}_{2}$ nor $1^{3} \mathrm{~A}_{1}$ states. Rather, the only important intersystem crossing channel is $1^{1} \mathrm{~A}_{2} \rightarrow 1^{3} \mathrm{~A}_{1}$ in a rate of $2.0 \mathrm{ps}, 10$ times slower than the rate obtained by Rai-Constapel and coworkers. ${ }^{22}$ The combined $1^{1} \mathrm{~A}_{2} \rightarrow$ triplets leads to a rate transfer of 1.5 ps. A similar rate is obtained if the dynamics are started directly from the $1^{1} \mathrm{~A}_{2}$ state (see $\mathrm{S} 8$ in Supporting information). Finally, the transfer between $1^{3} \mathrm{~A}_{1} \rightarrow 1^{3} \mathrm{~A}_{2}$ is fast, estimated at $602 / 803 \mathrm{fs}$ for the forth and back transfers (in ethanol, this was estimated at $12 \mathrm{ps).}{ }^{28}$ Solvents have a strong impact on the energetic order of $n \pi^{*}$ and $\pi \pi^{*}$ states of aromatic ketones, and this has a major impact on the dynamics in the excited state. Using the vacuum model, we have shifted the vertical energies to the values obtained for xanthone in solution (see Table $\mathrm{S} 2$ and Figure $\mathrm{S} 6$ in the Supporting Information). In water, the wavepacket stays trapped mainly on the singlet $1^{1} \mathrm{~A}_{2}$ state, while the $2^{3} \mathrm{~A}_{1}$ is the most populated among the triplets. In methanol, a slow build up of $1^{1} \mathrm{~A}_{2}$ state is observed, and triplet population is negligible. Still, solvent molecules can participate in the delocalization and

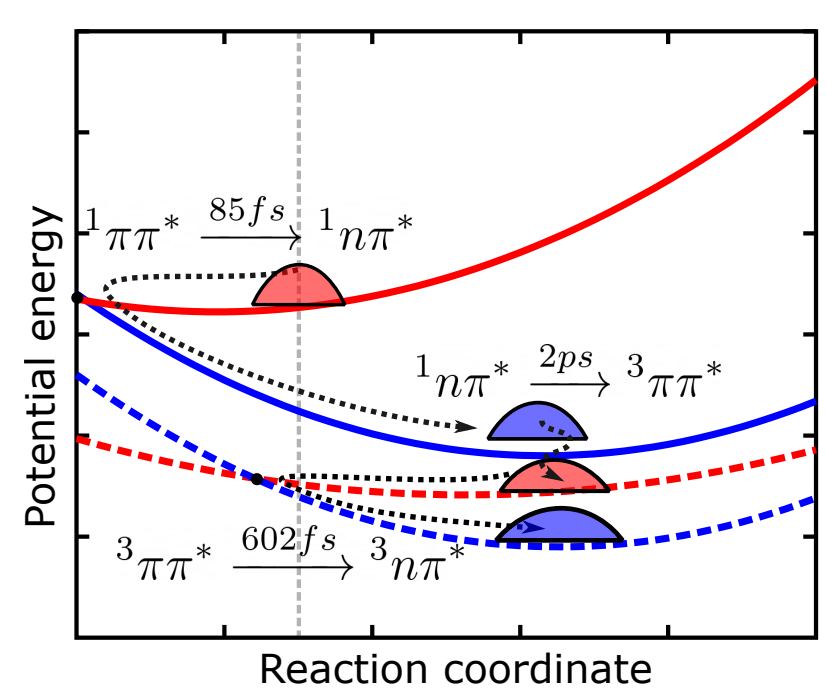

FIG. 4. Summary of the dynamical photochemical mechanism of xanthone in the gas phase starting $2^{1} \mathrm{~A}_{1}$ state as extracted from quantum dynamics.

orientation of carbonyl's lone pair. Therefore, more general models containing solvent molecules should be constructed to simulate the excited state dynamics of xanthone in solution.

To summarize, we have developed a vibronic model for xanthone including Duschinsky effects for describing equivalently the competition between internal conversion and intersystem crossing effects in xanthone. The reaction from the quantum dynamics is summarized in Fig. 4. We determine an internal conversion of $85 \mathrm{fs}$ from ${ }^{1} \pi \pi \rightarrow{ }^{1} n \pi^{*}$ state, followed by an intersystem crossing from ${ }^{1} n \pi^{*} \rightarrow{ }^{3} \pi \pi^{*}$ with a rate of $2.0 \mathrm{ps}$. We find no evidence of direct transfer from ${ }^{1} \pi \pi^{*}$ to the triplets. In conclusion, this dynamical model provides one of the most complete description of xanthone to date, and can be applied in the future to clarify the internal conversion kinetics of similar aromatic ketones.

\section{ACKNOWLEDGMENTS}

MHR acknowledges financial support by the Agence Nationale pour la Recherche through the project BIOMAGNET (ANR-16CE29-0008-01). Centre de Calcul Intensif d'Aix-Marseille is acknowledged for granting access to its high performance computing resources. MAR and CdG acknowledge the Spanish ministry of Science and Innovation (Project PID2020-113187GB-I00) and the Genralitat de Catalunya (project 2017-SGR629) for financial support.

\section{DATA AVAILABILITY}

The data that support the findings of this study are available from the corresponding author upon reasonable 
request.

${ }^{1}$ D. R. Kearns and W. A. Case, J. Am. Chem. Soc. 88, 5087 (1966).

${ }^{2}$ R. N. Griffin, Photochemistry and Photobiology 7, 159 (1968).

${ }^{3}$ D. E. Damschen, C. D. Merritt, D. L. Perry, G. W. Scott, and L. D. Talley, J. Phys. Chem. 82, 2268 (1978).

${ }^{4}$ G. Scharf and J. Winefordner, Talanta 33, 17 (1986).

${ }^{5}$ M. A. El-Sayed, J. Chem. Phys. 38, 2834 (1963).

${ }^{6}$ S. T. Park, J. S. Feenstra, and A. H. Zewail, J. Chem. Phys. 124, 174707 (2006).

${ }^{7}$ S. Aloïse, C. Ruckebusch, L. Blanchet, J. Réhault, G. Buntinx, and J.-P. Huvenne, J. Phys. Chem. A 112, 224 (2008).

${ }^{8}$ T. Kobayashi and S. Nagakura, Chem. Phys. Lett. 43, 429 (1976).

${ }^{9}$ G. Spighi, M.-A. Gaveau, J.-M. Mestdagh, L. Poisson, and B. Soep, Phys. Chem. Chem. Phys. 16, 9610 (2014).

${ }^{10}$ B. Soep, J.-M. Mestdagh, M. Briant, M.-A. Gaveau, and L. Poisson, Phys. Chem. Chem. Phys. 18, 22914 (2016).

${ }^{11}$ C.-W. Chang, T. I. Sølling, and E. W.-G. Diau, Chem. Phys. Lett. 686, 218 (2017).

12 J. C. Scaiano, J. Am. Chem. Soc. 102, 7747 (1980).

${ }^{13}$ J. J. Cavaleri, K. Prater, and R. M. Bowman, Chem. Phys. Lett. 259, 495 (1996).

${ }^{14}$ C. Ley, F. Morlet-Savary, J. Fouassier, and P. Jacques, J. Photochem. Photobiol. A 137, 87 (2000).

${ }^{15}$ C. Ley, F. Morlet-Savary, P. Jacques, and J. Fouassier, Chemical Physics 255, 335 (2000).

${ }^{16}$ I. Ghosh, A. Mukhopadhyay, A. L. Koner, S. Samanta, W. M. Nau, and J. N. Moorthy, Phys. Chem. Chem. Phys. 16, 16436 (2014).

${ }^{17}$ C. W. Ashpole, S. J. Formosinho, and G. Porter, Proceedings of the Royal Society of London. Series A, Mathematical and Physical Sciences 323, 11 (1971).

${ }^{18}$ N. S. Hill and M. L. Coote, J. Am. Chem. Soc. 140, 17800 (2018).

${ }^{19}$ S. Rani, J. Sobhanadri, and T. Prasada Rao, J. Photochem. Photobiol. A 94, 1 (1996).

${ }^{20}$ W. Domcke, D. Yarkony, and H. Koppel, eds., Conical Intersections: Theory, Computation and experiment, Advanced Series in Physical Chemistry, Vol. 17 (World Scientific Publishing Co Pte Ltd, 2011).

${ }^{21}$ N. Ohmori, T. Suzuki, and M. Ito, J. Phys. Chem. 92, 1086 (1988).
${ }^{22}$ V. Rai-Constapel, M. Etinski, and C. M. Marian, J. Phys. Chem. A 117, 3935 (2013).

${ }^{23}$ M. Huix-Rotllant, I. Burghardt, and N. Ferré, Comptes Rendus Chimie 19, 50 (2016).

${ }^{24}$ L. Favero, G. Granucci, and M. Persico, Phys. Chem. Chem. Phys. 18, 10499 (2016).

${ }^{25}$ V. Rai-Constapel, M. Kleinschmidt, S. Salzmann, L. SerranoAndrés, and C. M. Marian, Phys. Chem. Chem. Phys. 12, 9320 (2010).

${ }^{26}$ V. Rai-Constapel, S. Salzmann, and C. M. Marian, J. Phys. Chem. A 115, 8589 (2011).

${ }^{27}$ R. Mundt, T. Villnow, C. T. Ziegenbein, P. Gilch, C. Marian, and V. Rai-Constapel, Phys. Chem. Chem. Phys. 18, 6637 (2016).

${ }^{28}$ H. Satzger, B. Schmidt, C. Root, W. Zinth, B. Fierz, F. Krieger, T. Kiefhaber, and P. Gilch, J. Phys. Chem. A 108, 10072 (2004).

${ }^{29}$ B. Heinz, B. Schmidt, C. Root, H. Satzger, F. Milota, B. Fierz, T. Kiefhaber, W. Zinth, and P. Gilch, Phys. Chem. Chem. Phys. 8, $3432(2006)$.

${ }^{30}$ B. Heinz, B. Schmidt, C. Root, F. Milota, B. Fierz, T. Kiefhaber, W. Zinth, and P. Gilch, in Ultrafast Phenomena XV, edited by P. Corkum, D. M. Jonas, R. J. D. Miller, and A. M. Weiner (Springer Berlin Heidelberg, Berlin, Heidelberg, 2007) pp. 513515.

${ }^{31}$ M. Bracker, C. M. Marian, and M. Kleinschmidt, J. Chem. Phys. 155, 014102 (2021).

${ }^{32}$ T. E. Sharp and H. M. Rosenstock, J. Chem. Phys. 41, 3453 (1964).

${ }^{33}$ A. Thöny and M. J. Rossi, J. Photochem. Photobiol. A 104, 25 (1997).

${ }^{34}$ E. J. Heller, J. Chem. Phys. 68, 2066 (1978).

${ }^{35}$ V. Engel, Chem. Phys. Lett. 189, 76 (1992).

${ }^{36}$ F. Santoro, R. Improta, A. Lami, J. Bloino, and V. Barone, J. Chem. Phys. 126, 084509 (2007).

${ }^{37}$ F. Santoro, A. Lami, R. Improta, J. Bloino, and V. Barone, J. Chem. Phys. 128, 224311 (2008).

${ }^{38}$ M. Baba, T. Kamei, M. Kiritani, S. Yamauchi, and N. Hirota, Chem. Phys. Lett. 185, 354 (1991).

${ }^{39}$ Y. Ohshima, T. Fujii, T. Fujita, D. Inaba, and M. Baba, J. Phys. Chem. A 107, 8851 (2003). 